3 Norn MS. Preoperative trypan blue vital staining of corneal endothelium; eight years' follow up. Acta Ophthalmol 1980; 58: 550-555.

4 Pels E, Schuchand Y. Organ culture and endothelial evaluation as a preservative method in corneas. In: Bright bill FS (ed) Corneal Surgery; Theory, Technique and Tissue, Vol. 93 CV Mosby: St Louis, 1986 p. 102.

5 Bhartiya P, Sharma N, Ray M, Sinha R, Vajpayee RB. Trypan blue assisted phacoemulsification in corneal opacities. $\mathrm{Br} \mathrm{J}$ Ophthalmol 2002; 86: 857-859.

JS Titiyal, R Sinha, N Sharma and RB Vajpayee

Rajendra Prasad Centre for Ophthalmic Sciences, All India Institute of Medical Sciences, New Delhi-110029, India

Correspondence: JS Titiyal

Tel: 911126964817 ;

Fax: 911126588919.

E-mail: titiyal@rediffmail.com

Eye (2006) 20, 386-388. doi:10.1038/sj.eye.6701854; published online 4 March 2005

\section{Sir, \\ Conjunctival ischaemia subsequent to posterior subtenon's triamcinolone acetonide injection}

Triamcinolone acetonide is a potent corticosteroid frequently used for the treatment of a variety of ocular inflammatory conditions. Numerous complications have been reported. We report a case of conjunctival ischaemia following posterior subtenon's triamcinolone acetonide for diabetic macular oedema. To the best our knowledge this has not been previously reported.

\section{Case report}

A 58-year-old male patient was seen in the retinal clinic. He was diagnosed with diabetes 16 years ago and was subsequently diagnosed hypertensive. He had bilateral cataract extractions with lens implants in the early 1990s and his best-corrected visual acuities were 6/4 OD and 6/6 OS. His medication included insulin, ACE inhibitors, and aspirin. There was no history of allergic reactions. He had focal argon laser treatment for clinically significant macular oedema (CSMO) - once to the right and three times to the left eye over a period of 30 months. His vision deteriorated to 6/18 OS and the macular oedema persisted despite laser treatment. Fluorescein angiography revealed florid macular oedema with no

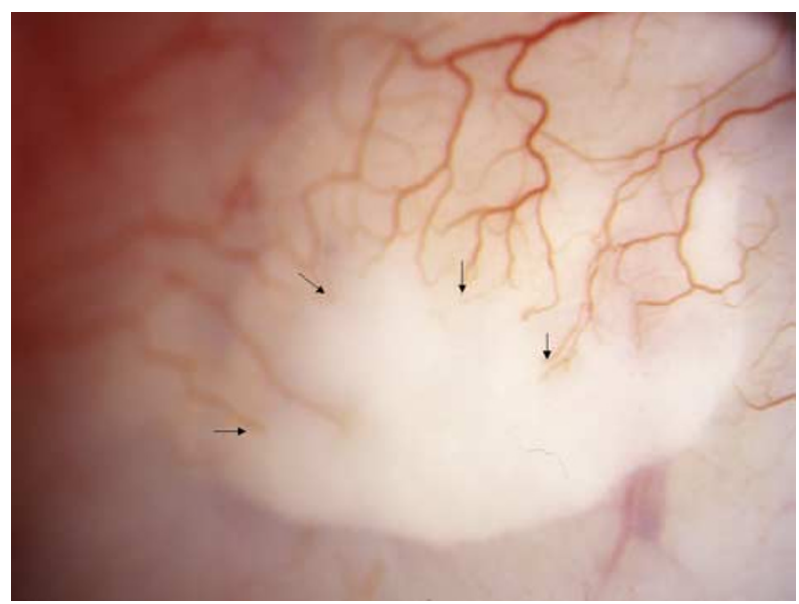

Figure 1 Subconjunctival deposit of triamcinolone superotemporally. Early reperfusion of conjuctival vessels 3 months postinjection (black arrows).

evidence of capillary nonperfusion. In all, $40 \mathrm{mg}$ of triamcinolone acetonide was injected into the posterior subtenon's space superotemporally. At 2 weeks after the injection, a deposit of triamcinolone located anteriorly under the conjunctiva was evident (Figure 1). The overlying conjunctival vessels were obliterated, and showed no sign of perfusion until 3 months after the injection, when larger vessels were noted to appear superiorly (Figure 1). His vision has remained stable at $6 / 18$ OS, with nonresolution of the macular oedema.

\section{Comment}

High-dose periocular corticosteroid injections are routinely used for the treatment of numerous inflammatory eye conditions. ${ }^{1-4}$ They are commonly used in the management of intermediate or posterior uveitis and cystoid macular oedema. Different modes of administration of the injection have been described - orbital floor, ${ }^{4}$ posterior subtenon's and transtenon's retrobulbar infusions. $^{3}$ The aim of these periocular injections is to deliver high doses of the drug locally, while reducing to a minimum unwanted systemic side effects. However, local complications do occur and many have been described - including: raised intraocular pressure, cataract progression, ptosis, retrobulbar haemorrhage, subconjuctival haemorrhage, globe perforation, retinal and choroidal vascular occlusions, and conjuctival ulceration. ${ }^{1-4}$

The patient we describe had a localized collection of subconjunctival triamcinolone, associated with conjunctival vasculature nonperfusion. Glucocorticoids have been well documented to potentiate the vasoconstrictive effects of circulating catecholomines, ${ }^{5}$ which may be responsible for this area of focal 
conjunctival ischaemia. Another possibility could be a localized toxic reaction to the triamcinolone acetonide. At 3 months subsequent to the injection there appears to be superior reperfusion over this area, but the vessels appear larger than the surrounding normal conjunctival vasculature. No related complications have arisen from this.

\section{Acknowledgements}

Proprietary interest/Financial interest: None.

\section{References}

1 Agrawal S, Agrawal J, Agrawal TP. Conjunctival ulceration following triamcinolone injection. Am J Ophthalmol 2003; 136: 539-540.

2 Helm CJ, Holland GN. The effects of posterior subtenon injection of triamcinolone acetonide in patients with intermediate uveitis. Am J Ophthamol 1995; 120: 55-64.

3 Okada AA, Wakabayashi T, Morimura Y, Kawahara S, Kojima E, Asano Y et al. Trans-tenon's retrobulbar triamcinolone infusion for the treatment of uveitis. $\mathrm{Br} J$ Ophthalmol 2003; 87: 968-971.

4 Riordan-Eva P, Lightman S. Orbital floor steroid injections in the treatment of uveitis. Eye 1994; 8: 66-69.

5 Yang S, Zhang L. Glucocorticoids and vascular reactivity. Curr Vasc Pharmacol 2004; 2(1): 1-12.

A Rubinstein'1, RJ Hanson², SDM Chen², N Porter ${ }^{2}$ and SM Downes ${ }^{2}$

${ }^{1}$ Ophthalmology Department, King Edward VII

Hospital, Windsor, Berkshire, UK

${ }^{2}$ Oxford Eye Hospital, Oxford, UK

Correspondence: A Rubinstein,

Ophthalmology Department,

King Edward VII Hospital, Windsor,

Berkshire SL4 3DP, UK

Tel: + 441753860441 ;

Fax: + 441753636487.

E-mail: arubinstein@btinternet.co.uk

Eye (2006) 20, 388-389. doi:10.1038/sj.eye.6701860;

published online 1 April 2005

Sir,

Therapeutic corneal tattoo following peripheral iridotomy complication

Occludable narrow angles predispose to angle closure glaucoma and merit prophylactic peripheral iridotomy (PI). We highlight the prevention of glare by careful placement of PIs and secondly, an unreported therapeutic indication of corneal tattooing for relief of such symptoms in patients with phakic narrow angle glaucoma (NAG).

\section{Case report}

An asymptomatic 59-year-old lady, diagnosed with chronic NAG, underwent bilateral YAG laser PI at her local ophthalmic department. She developed troublesome glare from each eye immediately afterwards. Her two sisters and mother were also diagnosed with chronic NAG. After 10 months her symptoms persisted and she was referred for a second opinion.

Her visual acuity was $6 / 6$ bilaterally. She had clear corneas and lenses bilaterally and no glaucomflecken. The brown irides showed no spiraling but had large laser iridotomies at $10 \mathrm{o}^{\prime}$ clock in the right eye and 2 o'clock in the left. Intraocular pressure was $14 \mathrm{mmHg}$ in both eyes and both optic discs were healthy with a cup disc ratio of 0.2 . Gonioscopy showed bilateral open inferior quadrants and nasal appositional closure with evidence of past closure in superior quadrant: sawtooth peripheral anterior synaechiae to midpoint of superior angle trabecular meshwork (TM) and blotchy pigment on the TM. The PIs were placed exactly where the upper lid margin joins the tear meniscus (Figure 1a and b).

She could not tolerate peripheral opaque contact lenses, which provided complete relief of symptoms. ${ }^{1}$ She underwent corneal tattooing, under topical anaesthesia. Filter paper, soaked with sterile platinum chloride solution, was inserted into a $2 \mathrm{~mm}$ corneal stromal pocket, created with a keratome, anterior to the PI. The reducing agent, hydrazine hydrate solution, was then slowly added beneath the filter paper. Excess solution was soaked up by limbal placed dry swabs to minimise conjunctival staining (Figure 1c and d). Symptomatic relief from glare was achieved, without ocular inflammation or corneal erosion symptoms at 20 months follow up.

\section{Comment}

Glare occurred from the acquired peripheral iris defects, particularly, at the level of the upper lid margin-tear film interface. ${ }^{2}$ Gonioscopic findings suggested that she had occludable angles, which did merit bilateral prophylactic laser PIs. She declined bilateral upper eyelid lowering, for cosmetic reasons. Intraocular iris suturing to close the PIs was an option but ran the risk of hyphaema, uveitis, and an increased risk of the development of glaucoma and cataract. Therefore, she was offered corneal tattooing.

A corneal stromal pocket (Figure 1c), by keratome, minimised reported complications of corneal tattooing 